\title{
On the difficulty of task switching: Assessing the role of task-set inhibition
}

\author{
MEI-CHING LIEN \\ Oregon State University, Corvallis, Oregon \\ ERIC RUTHRUFF \\ University of New Mexico, Albuquerque, New Mexico \\ and \\ DAVID KUHNS \\ Oregon State University, Corvallis, Oregon
}

\begin{abstract}
This study assessed whether or not the difficulty of task switching stems from previous inhibition of the task set. A predictable sequence of univalent stimuli (affording performance of one active task) and bivalent stimuli (affording performance of two tasks) was used in two experiments. Experiment 1 used an alternating-runs paradigm $(\mathrm{AABB})$ and Experiment 2 used a strictly alternating sequence (ABAB). The critical variable was whether the incentive for task-set inhibition was strong (on bivalent trials) or weak (on univalent trials). The question was whether it would be more difficult to switch to a task that previously needed to be inhibited than to a task that did not need to be inhibited. This pattern was not observed in either experiment. Thus, the data provide no evidence that task switching is difficult because of the need to overcome recent task-set inhibition.
\end{abstract}

In everyday life, people often have many different tasks pending at the same time. People can flexibly switch back and forth between these tasks even when the impetus to switch tasks is purely top-down (goal driven), without support from any environmental stimuli. Nevertheless, people usually cannot do so without cost. Numerous studies have shown that performance slows immediately following a task switch (known as the switch cost, Jersild, 1927). Switch costs occur even when people know the identity of the new task in advance (see, e.g., Lien, Schweickert, $\&$ Proctor, 2003; Sohn \& Carlson, 2000) and have ample time to prepare for the switch (see, e.g., Allport, Styles, \& Hsieh, 1994; Meiran, 1996; Rogers \& Monsell, 1995). These findings raise two important questions: What cognitive processes enable flexible task switching and why do these processes slow subsequent task performance?

Several recent studies have suggested that the cognitive processes involved in task switching include inhibition of the previous task set (Mayr \& Keele, 2000; see also Hübner, Dreisbach, Haider, \& Kluwe, 2003; Schuch \& Koch, 2003). In Mayr and Keele (2000), participants viewed

This research was supported by funding from NASA Grant NCC 21325 to M.-C.L., the Oregon Space Grant Consortium Graduate Fellowship to D.K., and the Human Measures and Performance Project of NASA's Airspace Systems Program. We thank Rich Carlson, Mike Wendt, Stefanie Schuch, Collin Green, and Joel Lachter for helpful comments on earlier drafts of this article. Correspondence concerning this article should be addressed to M.-C. Lien, Department of Psychology, Oregon State University, Corvallis, OR 97331-5303 (e-mail: mei.lien@ oregonstate.edu). four rectangular objects that could differ on three dimensions (color, orientation, and movement). A visual word cue ("Color," "Orientation," or "Movement") instructed participants to respond to the deviant object on that dimension. To look for evidence of task-set inhibition, Mayr and Keele compared ABA and CBA task sequences. They reasoned that task-set inhibition would decay over time, so a switch to Task A would be slower if that task had been inhibited recently (e.g., as in ABA) than if it had not been (as in CBA). The data confirmed this prediction.

Task-set inhibition can have positive effects (facilitating an immediate switch to a new task) as well as negative effects (impeding a subsequent switch back to the inhibited task). Thus, task-set inhibition can explain not only how people successfully switch tasks, but also why task-switch performance is slow. Furthermore, it can explain why switch costs are much larger with bivalent stimuli, affording performance of two active tasks, than with univalent stimuli, affording performance of only one active task.

First, consider bivalent stimuli. When performing a letter task (vowel/consonant) and a digit task (odd/even), for instance, participants might see both a letter and a digit on each trial. Because the stimulus display affords both tasks, there is a serious risk of performing the irrelevant task and making an error. The risk would be especially severe on task-switch trials, because the irrelevant task is the one that was just performed. To prevent task perseveration errors on switch trials, one could inhibit the just-performed (now irrelevant) task. Note that although task-set inhibition would facilitate switching on the upcoming trial, it could impede switching back to that inhibited task on a 
future trial. Thus, inhibition can, by itself, explain why bivalent task switches (in which the task is inhibited) are performed much more slowly than bivalent task repetitions (in which the task is not inhibited).

Now consider univalent stimuli (e.g., when the display contains only a letter or only a digit). Because the Task A stimulus does not afford Task B, there is no risk of accidentally performing Task B and therefore little incentive to inhibit Task B (even when it was just performed; see Fagot, 1994). The absence of inhibition could explain why people can switch between univalent tasks with very little cost (see, e.g., Allport et al., 1994; Jersild, 1927; Ruthruff, Remington, \& Johnston, 2001; Spector \& Biederman, 1976).

A key assumption of this explanation of bivalent and univalent switch costs is that task-set inhibition is applied adaptively, to prevent performance of the wrong task. Inhibition is unnecessary when it is known that the task will repeat or that the stimulus will be univalent. We also assume, following Mayr and Keele (2000), that inhibition is applied proactively, in anticipation of bivalence, although similar predictions follow if inhibition is also applied reactively, in response to bivalence. This adaptive view of task-set inhibition is simple, plausible, and consistent with previous results. At the same time, this hypothesis has not yet been tested rigorously.

\section{The Present Study}

The purpose of the present study was to evaluate the adaptive task-set inhibition hypothesis, which assumes that task switching is difficult primarily because of the need to overcome residual task-set inhibition. Mayr and Keele (2000) found that mean response time (RT) was roughly $40 \mathrm{msec}$ faster on the third trial of a CBA task sequence than it was on an ABA task sequence. They attributed this effect to the extra decay of Task A inhibition in the CBA sequence compared with the ABA sequence. These results, however, do not indicate whether or not switch costs are primarily due to task-set inhibition. On the one hand, the observed effect seems small compared with the typical switch cost $(\sim 200 \mathrm{msec})$. On the other hand, it is not clear how rapidly inhibition decays over time, or even that it decays (the need to ignore the irrelevant task on intervening trials could actually increase inhibition; see Experiment 2 of Mayr \& Keele, 2000). If both the ABA and CBA conditions have similar degrees of residual inhibition, the observed effect size would greatly underestimate the true cost of residual inhibition.

Rather than compare conditions differing only in the recency of inhibition (e.g., as in Mayr \& Keele, 2000), we compared two conditions differing in the need for inhibition. Consider an ABA task sequence. After one performs the first instance of Task $\mathrm{A}$, there is a strong incentive to inhibit that task set when the intervening Task B stimulus is bivalent (to prevent perseveration of Task A). The need to overcome residual inhibition would then slow the subsequent switch back to Task A, provided that the inhibition cannot be completely counteracted by top-down mechanisms and does not completely dissipate during the intertrial interval. In contrast, there would appear to be very little incentive to inhibit Task A when the intervening Task B stimulus is univalent and perseveration of Task A is impossible. The lack of inhibition would then facilitate the subsequent switch back to Task A.

In summary, the difficulty of switching back to Task A in an ABA sequence should depend critically on whether the preceding Task B stimulus display was bivalent or univalent (for a similar logic, see Allport \& Wylie, 1999). Experiments 1 and 2 used different approaches to test this prediction. Note that many previous studies have manipulated univalence and bivalence, but they have generally done so between blocks, confounding current univalence/ bivalence and previous univalence/bivalence (for a few exceptions, see Fagot, 1994, and Rogers \& Monsell, 1995).

\section{EXPERIMENT 1}

Experiment 1 used a variant of the traditional alternatingruns paradigm (AABB). Task $\mathrm{A}$ was a digit task (odd vs. even) and Task B was a letter task (vowel vs. consonant), or vice versa (counterbalanced across participants). When Task A was performed, the stimulus was always bivalent (a letter and a digit), but when Task B was performed, the stimulus was always univalent (a letter or a digit). We denote this sequence as $A_{B} A_{B} B B$, where the subscript $B$ indicates that an irrelevant Task $B$ stimulus was presented alongside the relevant Task A stimulus. The use of a predictable task sequence makes it easy for participants to adapt to the univalence/bivalence of the displays. To further encourage adaptation, the participants were informed of this design and were given two practice blocks.

When switching from Task $B$ to Task $A_{B}$, participants have strong incentive to inhibit Task B (to avoid task perseveration). This inhibition could produce a cost when later switching back to Task B (e.g., in the third trial in the sequence $A_{B} A_{B} \underline{B} B$ ), even though the stimulus for Task $B$ is univalent. In contrast, there is no incentive to inhibit Task A when switching to the univalent Task B. If Task $\mathrm{A}$ is not inhibited, then it should be relatively easy to switch back to Task $A_{B}$ (e.g., the first trial in the sequence $\underline{A}_{B} A_{B} B B$ ), even though the stimulus for Task $A_{B}$ is bivalent.

In summary, the strong version of the adaptive inhibition hypothesis predicts that the difficulty of switching to a task should depend critically on whether the preceding trials were univalent or bivalent (manipulating the incentive for inhibition), not whether the current trial is univalent or bivalent. Because previous studies have generally confounded previous and current univalence/bivalence, they cannot address this issue. One notable exception involves the work of Wylie and Allport (2000, Experiment 1; also discussed in Allport \& Wylie, 1999), who conducted an alternating-runs experiment with Stroop tasks (color naming and word naming). Interestingly, their results generally confirmed the above predictions: They found smaller switch costs following univalent trials than bivalent trials. These results, although suggestive, should be interpreted cautiously. One reason is that the key 
univalence/bivalence manipulation was between, rather than within, blocks. Another reason is that switching between asymmetric tasks may represent a special case (we will return to these issues in the General Discussion).

\section{Method}

Participants. A total of 32 undergraduates at Oregon State University participated in exchange for extra course credit. All had normal or corrected-to-normal vision.

Apparatus and Stimuli. Stimuli were presented on IBMcompatible microcomputers connected to 21 -in. Dell monitors. An $11.4 \mathrm{~cm} \times 11.4 \mathrm{~cm}$ frame consisting of four boxes was presented in the center of the screen (see Figure 1). On each trial, stimuli $(0.8 \mathrm{~cm}$ wide $\times 0.9 \mathrm{~cm}$ tall) appeared in one of these boxes. At an average viewing distance of $55 \mathrm{~cm}$, each stimulus subtended a visual angle of $0.83^{\circ} \times 0.94^{\circ}$

Design and Procedure. On each trial, the participants performed either a digit task or a letter task. For the digit task, the participants pressed the Z key for odd digits $(1,3,7$, or 9) and the M key for even digits $(2,4,6$, or 8$)$. For the letter task, the participants pressed the $\mathrm{Z}$ key for consonants $(\mathrm{G}, \mathrm{K}, \mathrm{M}$, or R) and the $\mathrm{M}$ key for vowels (A, $\mathrm{E}, \mathrm{I}$, or $\mathrm{U})$. The participants pressed the $\mathrm{Z}$ and $\mathrm{M}$ keys with their left index and right index fingers, respectively.

Following Rogers and Monsell (1995), the first stimulus of each block appeared in the top-left box (see Figure 1). Each subsequent stimulus appeared in the box located immediately clockwise from the previous one. Each trial started with a fixation cross, which lasted for $500 \mathrm{msec}$. After a 300-msec blank period, the stimulus appeared. After the participants responded, auditory feedback (a beep on error trials, silence on correct trials) was presented for $200 \mathrm{msec}$. The fixation cross for the next trial appeared $400 \mathrm{msec}$ later. Consequently, the total response-stimulus interval was $1,400 \mathrm{msec}$.

For the top two boxes (the bivalent stimulus condition), a digit and a letter appeared simultaneously in the center of the box. As shown in Figure 1, these stimuli were presented in a horizontal row; the relative positions of the relevant and irrelevant stimuli were determined randomly. The identity of the relevant stimulus was chosen randomly, with the restriction that each category (odd, even, consonant, vowel) be used equally often within each block. The identity of the irrelevant stimulus was selected randomly, without any restriction. For the bottom two boxes (the univalent stimulus condition), a single stimulus appeared in the center of the box. For half of the participants, the top two boxes were assigned to the digit task and the bottom two boxes were assigned to the letter task (producing a task sequence of digit-digit-letter-letter... ). For the other participants, the assignment was reversed.

The participants performed two practice blocks; the first contained 16 trials and the second contained 64 trials. The following six experimental blocks each contained 64 trials. The entire experiment lasted approximately $25 \mathrm{~min}$. Both speed and accuracy were emphasized. After each block, the participants received feedback regarding their mean RT and accuracy for that block. They were encouraged to rest before beginning the next block.

\section{Results}

We omitted from analysis any task repetition trial in which the stimulus was the same as the previous stimulus, to avoid contamination of task repetition effects with stimulus repetition effects. We also omitted trials following an error. For RT analyses, we also omitted error trials. In addition, we treated RTs outside the range of $100 \mathrm{msec}$ to $4,000 \mathrm{msec}$ as outliers, eliminating an additional $0.2 \%$ of trials. Because task type (letter vs. digit) had little effect and did not consistently interact with any other factors, it was excluded from the final analyses. Data were analyzed as a function of task transition (repetition vs. switch) and previous trial type (univalent vs. bivalent).

Table 1 shows the mean RT and proportion of error (PE) in each condition. For the RT data, there was a main effect of task transition $\left[F(1,31)=43.69, M S_{\mathrm{e}}=11,031, p<.001\right]$, reflecting an overall switch cost of $124 \mathrm{msec}$. There was also a main effect of previous trial type $[F(1,31)=47.04$, $\left.M S_{\mathrm{e}}=9,736, p<.001\right]$; mean RT was $120 \mathrm{msec}$ shorter when the previous trial was bivalent $(M=611 \mathrm{msec})$ than when it was univalent $(M=731 \mathrm{msec})$. The interaction between task transition and previous trial type was also significant $\left[F(1,31)=10.56, M S_{\mathrm{e}}=1,965, p<.01\right]$; switch costs on RT were larger when the previous trial was univalent $(148 \mathrm{msec})$ than when the previous trial was bivalent (98 $\mathrm{msec})$.

For the PE data, there was a main effect of task transition $\left[F(1,31)=7.74, M S_{\mathrm{e}}=0.0010, p<.01\right]$; the switch cost on PE was .016. No other effects were significant.

\section{Discussion}

If adaptive inhibition is the primary source of switch costs, then it should be more difficult to switch back to a task when the preceding trials were bivalent (requiring inhibition) than when they were univalent (not requiring inhibition). The results actually went in the opposite direction: Mean RT on switch trials was $145 \mathrm{msec}$ faster $(S E=$ $22 \mathrm{msec})$ following bivalent trials $(M=660 \mathrm{msec})$ than following univalent trials $(M=805 \mathrm{msec})$.

These results suggest that something other than adaptive inhibition is the primary source of switch costs (we offer a few hypotheses in the General Discussion). However, the

\section{Bivalent Stimulus Condition}

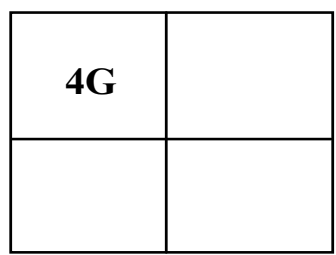

Univalent Stimulus Condition

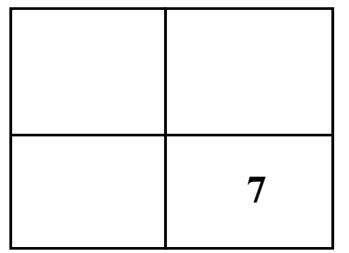

Figure 1. An example of the bivalent stimulus condition and the univalent stimulus condition used in Experiment 1. 
Table 1

Mean Response Times (RTs, in Milliseconds) and Proportions of Errors in Experiment 1 As a Function of Previous Trial

Type (Univalent or Bivalent) and Task Transition (Task Repetition or Task Switch)

\begin{tabular}{|c|c|c|c|c|c|c|}
\hline \multirow[b]{3}{*}{ Previous Trial } & \multicolumn{4}{|c|}{ Task Transition } & & \\
\hline & \multicolumn{2}{|c|}{ Repetition } & \multicolumn{2}{|c|}{ Switch } & \multicolumn{2}{|c|}{ Average } \\
\hline & RT & PE & RT & PE & RT & PE \\
\hline \multirow[t]{2}{*}{ Univalent } & 657 & .045 & 805 & .060 & 731 & .030 \\
\hline & \multicolumn{2}{|c|}{$\mathrm{A}_{\mathrm{B}} \mathrm{A}_{\mathrm{B}} \mathrm{B} \underline{\mathbf{B}}$} & \multicolumn{2}{|c|}{$\underline{\mathbf{A}}_{\underline{\mathbf{B}}} \mathrm{A}_{\mathrm{B}} \mathrm{BB}$} & & \\
\hline \multirow[t]{2}{*}{ Bivalent } & 562 & .046 & 660 & .062 & 611 & .054 \\
\hline & \multicolumn{2}{|c|}{$\mathrm{A}_{\mathrm{B}} \underline{\mathbf{A}}_{\underline{\mathbf{B}}} \mathrm{BB}$} & \multicolumn{2}{|c|}{$\mathrm{A}_{\mathrm{B}} \mathrm{A}_{\mathrm{B}} \underline{\mathrm{B} B}$} & & \\
\hline
\end{tabular}

Note-The task sequence used in Experiment 1 was $A_{B} A_{B} B B$, where the subscript $B$ indicates that an irrelevant Task $B$ stimulus was presented alongside the relevant Task A stimulus. In each cell of the table, the relevant trial within the task sequence is boldface and underlined. RT, response time; PE, proportion of errors.

results do not necessarily indicate that switch performance is completely insensitive to whether the previous trial was univalent or bivalent (manipulating the need for inhibition). Such effects might have occurred in this experiment but were outweighed by the effects of whether the current trial was univalent or bivalent. To evaluate this possibility, in Experiment 2, we independently manipulated previous univalence/bivalence and current univalence/bivalence.

\section{EXPERIMENT 2}

Whereas we used a repeating $A_{B} A_{B} B B$ task sequence in Experiment 1, we used a repeating $A_{B} B_{A} A B$ task sequence in Experiment 2. Thus, all trials were task-switch trials. One consequence of shortening the task run length is that there was less time for inhibition to decay between instances of a task. More importantly, this design allowed us to factorially manipulate whether the current trial was univalent or bivalent and whether the previous trial was univalent or bivalent. Another methodological difference is that we counterbalanced across participants whether the top two boxes were bivalent and the bottom two univalent (as in Experiment 1) or vice versa.

According to the adaptive inhibition hypothesis, taskswitch responses should be relatively slow when the preceding trial is bivalent (e.g., from $A_{B}$ to $B_{A}$ or from $B_{A}$ to $A$ ), because participants are switching to a recently inhibited task. Meanwhile, switch performance should be relatively fast when the preceding trial is univalent (e.g., from $A$ to $\mathrm{B}$ or from $\mathrm{B}$ to $\mathrm{A}_{\mathrm{B}}$ ), because participants are switching to a task that was not recently inhibited. In other words, taskswitch performance should depend strongly on whether the previous trial is univalent or bivalent, regardless of whether the current trial is univalent or bivalent.

\section{Method}

Participants. There were 36 participants, drawn from the same participant pool as in Experiment 1. None had participated in Experiment 1.

Apparatus, Stimuli, and Procedure. The apparatus, stimuli, and procedure were the same as those in Experiment 1, except that the participants always switched tasks (e.g., $\left.A_{B} B_{A} A B\right)$. The assign- ment of tasks (digit vs. letter) to the top-left box was counterbalanced across participants, as was the assignment of univalence/bivalence to the top and bottom boxes.

\section{Results}

The data analysis was similar to that of Experiment 1. Application of RT cutoffs eliminated $0.3 \%$ of responses. Data were analyzed as a function of current univalence/ bivalence and previous univalence/bivalence.

Table 2 shows the mean RT and PE. For the RT data, there was a main effect of current univalence/bivalence $\left[F(1,35)=215.65, M S_{\mathrm{e}}=13,776, p<.001\right]$; responses were $287 \mathrm{msec}$ slower when the current trial was bivalent than when it was univalent. The effect of previous univalence/bivalence was also significant $[F(1,35)=17.61$, $\left.M S_{\mathrm{e}}=5,162, p<.001\right]$. Contrary to the predictions of the adaptive inhibition hypothesis, however, responses were $51 \mathrm{msec}$ faster $(S E=12 \mathrm{msec})$ when the previous trial was bivalent than when it was univalent. The interaction between current univalence/bivalence and previous univalence/bivalence was also significant $[F(1,35)=$ $\left.16.50, M S_{\mathrm{e}}=12,048, p<.001\right]$. Follow-up data analyses revealed that previous univalence/bivalence had a significant effect when the current trial was bivalent $[F(1,35)=$ $\left.23.26, M S_{\mathrm{e}}=12,006, p<.001\right]$, but not when it was univalent $\left[F(1,35)=2.00, M S_{\mathrm{e}}=5,204, p=.1662\right]$.

For the PE data, there was only a main effect of current univalence/bivalence $\left[F(1,35)=11.78, M S_{\mathrm{e}}=0.0009\right.$, $p<.01$ ]; PE was .017 higher when the current trial was bivalent than when it was univalent.

\section{Discussion}

In Experiment 2, we independently manipulated whether the current trial was univalent or bivalent and whether the previous trial was univalent or bivalent, using a repeating $\mathrm{A}_{\mathrm{B}} \mathrm{B}_{\mathrm{A}} \mathrm{AB}$ task sequence. According to the adaptive inhibition hypothesis, task-switch performance should be better when the previous trial was univalent (little incentive for inhibition) than when the previous trial was bivalent (strong incentive for inhibition). The results, however,

Table 2

Mean Response Times (RTs, in Milliseconds) and Proportions of Errors in Experiment 2 As a Function of Whether Previous Trial Was Univalent or Bivalent and Whether Current Trial Was Univalent or Bivalent

\begin{tabular}{|c|c|c|c|c|c|c|}
\hline \multirow[b]{3}{*}{ Previous Trial } & \multicolumn{4}{|c|}{ Current Trial } & & \\
\hline & \multicolumn{2}{|c|}{ Univalent } & \multicolumn{2}{|c|}{ Bivalent } & \multicolumn{2}{|c|}{ Average } \\
\hline & RT & PE & RT & PE & RT & PE \\
\hline Univalent & $\begin{array}{r}721 \\
A_{1}\end{array}$ & $\begin{array}{l}.039 \\
\mathbf{A} \underline{\mathbf{B}}\end{array}$ & $\begin{array}{r}1,082 \\
\underline{\mathbf{A}}_{\mathbf{B}}\end{array}$ & $\begin{array}{l}.054 \\
\mathrm{~B}\end{array}$ & 902 & .047 \\
\hline Bivalent & $\begin{array}{r}745 \\
\mathrm{~A}\end{array}$ & $\begin{array}{l}.036 \\
\mathrm{~A} B\end{array}$ & $\begin{array}{r}958 \\
A_{B}\end{array}$ & $\begin{array}{l}.055 \\
\mathrm{~B}\end{array}$ & 852 & .046 \\
\hline Average & 733 & .038 & 1,020 & .055 & & \\
\hline
\end{tabular}

Note-The task sequence used in Experiment 2 was $A_{B} B_{A} A B$, where the subscript indicates that a stimulus from the irrelevant task was presented alongside the relevant stimulus. In each cell of the table, the relevant trial within the task sequence is boldface and underlined. RT, response time; $\mathrm{PE}$, proportion of errors. 
failed to confirm this prediction. If anything, the trend went in the opposite direction.

Although we found little effect of previous univalence/ bivalence, we found a very large main effect of current univalence/bivalence. In fact, RT was $287 \mathrm{msec}$ longer and PE was .017 higher when the current trial was bivalent rather than univalent. Note that participants responded very quickly and accurately to univalent task switches even when that task was assumed to have been recently inhibited (because the previous trial was bivalent).

\section{GENERAL DISCUSSION}

The present experiments investigated the hypothesis that switch costs occur because of the need to overcome previous inhibition. If inhibition is adaptive, people should not intentionally inhibit a task when there is no risk of a task perseveration error. This release from inhibition should benefit performance when it comes time to switch back to that task. Consequently, task-switch performance should be better following univalent trials (little incentive to inhibit the irrelevant task) than following bivalent trials (strong incentive to inhibit the irrelevant task).

To evaluate these predictions, we used an $A_{B} A_{B} B B$ task sequence in Experiment 1 and an $\mathrm{A}_{\mathrm{B}} \mathrm{B}_{\mathrm{A}} \mathrm{AB}$ task sequence in Experiment 2. Both experiments failed to confirm the predictions. If anything, the results went in the opposite direction. Thus, we found no evidence that adaptive taskset inhibition is the primary cause of, or even contributes to, switch costs.

The present findings appear to conflict with those of Wylie and Allport (2000; Experiment 1), who had participants switch between the color-naming and word-naming versions of the Stroop task. A key finding was that the cost of switching to the word-naming task (whose stimulus was always bivalent) was greater when the stimulus for the color-naming task was bivalent (a colored word) rather than univalent (a row of $X \mathrm{~s}$ ). This finding supports the hypothesis that inhibition of word naming increases when there is a strong need for it. One plausible reconciliation, therefore, is that inhibition is applied when it is necessary to prevent a dominant task from overwhelming a weaker task, as in Wylie and Allport, but not when the active tasks are roughly equal in strength (as in our study). It may be premature, however, to conclude that inhibition took place in Wylie and Allport's study. Rather than increasing inhibition of the word-naming task in the all-bivalent blocks, participants might have increased activation of the colornaming task. This heightened activation would impede a switch back to the word-naming task, thus explaining the key result without appealing to the concept of inhibition (see also Monsell, Yeung, \& Azuma, 2000). This ambiguity of interpretation stems from the fact that Wylie and Allport used only bivalent stimuli in word-naming trials; had they used univalent word-naming trials, they could potentially have deconfounded the contributions of wordnaming inhibition and color-naming activation. ${ }^{1}$ This issue deserves further investigation.

\section{Is Inhibition Triggered by Response Competition?}

Goschke (2000; see also Mayr \& Keele, 2000) proposed that inhibition might be more necessary when the irrelevant task suggests a response that is incongruent, rather than congruent, with the relevant-task response. Consistent with this claim, Goschke found somewhat larger switch costs following incongruent trials than congruent trials (roughly, $200 \mathrm{msec}$ vs. $150 \mathrm{msec}$ at the long SOA, estimating from his Figure 4.2). Note that Goschke assumed that inhibition is applied reactively, after detection of response competition. In contrast, we allow that inhibition might also be applied proactively, in anticipation of potential competition.

Nevertheless, to see if similar effects occurred in the present experiments, we measured the amount of slowing following an incongruent bivalent stimulus compared with a congruent bivalent stimulus. In Experiment 1, the slowing was $-3 \mathrm{msec}$ when the current trial was univalent and $-1 \mathrm{msec}$ when it was bivalent; neither effect was significant $[F \mathrm{~s}(1,31)<1.0]$. In Experiment 2, the slowing was $11 \mathrm{msec}$ on univalent trials and $32 \mathrm{msec}$ on bivalent trials; again, neither effect was statistically significant $\left[F(1,35)<1.0\right.$ and $F(1,35)=3.29, M S_{\mathrm{e}}=11,093, p=$ $.08]$, respectively. The difference in the congruence effects between the univalent trials and the bivalent trials was also not statistically significant $[F(1,35)<1.0]$. To summarize, the amount of extra slowing following incongruent trials was negligible in three out of four comparisons. Even when a hint of slowing was observed, it was difficult to determine conclusively that it was due to heightened inhibition, rather than some other influence. For instance, incongruence on one trial might lead to more cautious responding on subsequent trials. Overall, we found little evidence that extra task-set inhibition is triggered by an incongruent irrelevant-task response.

\section{Why Doesn't Adaptive Inhibition Lead to Switch Costs?}

One straightforward explanation of these findings is that people do not inhibit task sets. In other words, task switching might be facilitated primarily by activating the relevant task set rather than by inhibiting the irrelevant task set. The residual activation of the irrelevant task on switch trials would slow performance and produce a switch cost (at least on bivalent trials).

Given the strong intuitive appeal of the concept of taskset inhibition, and previous evidence in favor of it (see, e.g., Hübner et al., 2003; Mayr \& Keele, 2000), it is worth considering how one might reconcile this core assumption with the present findings. One possibility is that task-set inhibition occurs, and does lead to future switch costs, but that it is not adaptive. People might inhibit task sets automatically, even when the immediate benefits are small and the future costs are large. Mayr and Keele (2000), for instance, suggested that task-set inhibition is controlled by "processes that are relatively impenetrable through higher-level control" (p. 22). Completely nonadaptive in- 
hibition seems implausible, because people presumably do not inhibit tasks expected to repeat on the next trial. It is conceivable, however, that people inhibit tasks whenever a switch is expected, regardless of the relative costs and benefits. This hypothesis appears to predict slow responses on task-switch trials (due to the costs of overcoming residual inhibition) even when the current stimulus is univalent. The present data clearly disconfirmed this prediction. To explain these findings, one would need the additional (and somewhat questionable) assumption that inhibition does not impair performance with univalent stimuli.

An alternative possibility is that inhibition occurs, and is adaptive, but does not produce switch costs. For instance, top-down preparation to switch to a previously inhibited task might completely override the inhibition (although previous studies have found no evidence that increasing preparation time decreases inhibition; see, e.g., Goschke, 2000; Mayr \& Keele, 2000). If lingering inhibition is not the cause of switch costs, then what is? One possibility is that switch costs occur because persisting activation of the irrelevant task (which Allport et al., 1994, called task set inertia) interferes with the execution of the relevant task. Another possibility (not necessarily incompatible with the first) is that switch costs occur because people cannot completely reconfigure a new task set in advance of a stimulus (Lien, Ruthruff, Remington, \& Johnston, 2005; Rogers \& Monsell, 1995).

Although these alternative hypotheses can explain why we failed to find faster responses following a univalent trial than following a bivalent trial, none of them provide an obvious explanation of why the effect actually went in the opposite direction when the current stimulus was bivalent. At this point, we can only speculate that univalent trials cause participants to "let down their guard" somewhat, so that they expend less effort on subsequent bivalent trials.

\section{Conclusions}

As in previous studies, we found that task-switch performance was very sensitive to whether the current stimulus was univalent or bivalent. However, we found that taskswitch performance was not sensitive to whether the previous trial was univalent or bivalent. In other words, performance of a task switch was no faster when the recent incentive to inhibit that task was weak rather than strong. These findings argue against the hypothesis that people inhibit tasks adaptively and that task switching is so difficult because of the need to overcome lingering inhibition. Instead, the results suggest three alternative hypotheses: (1) task-set inhibition is weak or nonexistent, (2) inhibition occurs but is not adaptive (i.e., is applied even when the cost/benefit ratio is unfavorable), or (3) inhibition occurs but can easily be removed before the task becomes relevant again. Further research is needed to test these hypotheses.

\section{REFERENCES}

Allport, D. A., Styles, E. A., \& Hsieh, S. (1994). Shifting intentional set: Exploring the dynamic control of tasks. In C. Umiltà and M. Moscovitch (Eds.), Attention and performance XV: Conscious and nonconscious information processing (pp. 421-452). Cambridge, MA: MIT Press.

Allport, [D.] A., \& Wylie, G. (1999). Task-switching: Positive and negative priming of task-set. In G. W. Humphreys, J. Duncan, \& A. Treisman (Eds.), Attention, space, and action: Studies in cognitive neuroscience (pp. 273-296). Oxford: Oxford University Press.

FAGOT, C. (1994). Chronometric investigations of task switching. Unpublished doctoral dissertation, University of California, San Diego. Retrieved April 2, 2002, from http://www.pashler.com/manuscripts.html.

GoschKe, T. (2000). Intentional reconfiguration and involuntary persistence in task set switching. In S. Monsell \& J. Driver (Eds.), Control of cognitive processes: Attention and performance XVIII (pp. 331-356). Cambridge, MA: MIT Press.

Hübner, M., Dreisbach, G., Haider, H., \& Kluwe, R. H. (2003). Backward inhibition as a means of sequential task-set control: Evidence for reduction of task competition. Journal of Experimental Psychology: Learning, Memory, \& Cognition, 29, 289-297.

Jersild, A. T. (1927). Mental set and shift. Archives of Psychology, 14 (Whole No. 89).

Lien, M.-C., Ruthruff, E., Remington, R. W., \& Johnston, J. C. (2005). On the limits of advance preparation for a task switch: Do people prepare all the task some of the time or some of the task all the time? Journal of Experimental Psychology: Human Perception \& Performance, 31, 299-315.

Lien, M.-C., Schweickert, R., \& Proctor, R. W. (2003). Task switching and response correspondence in the psychological refractory period paradigm. Journal of Experimental Psychology: Human Perception \& Performance, 29, 692-712.

Mayr, U., \& Keele, S. W. (2000). Changing internal constraints on action: The role of backward inhibition. Journal of Experimental Psychology: General, 129, 4-26.

Meiran, N. (1996). Reconfiguration of processing mode prior to task performance. Journal of Experimental Psychology: Learning, Memory, \& Cognition, 22, 1423-1442.

Monsell, S., Yeung, N., \& Azuma, R. (2000). Reconfiguration of task-set: Is it easier to switch to the weaker task? Psychological Research, 63, 250-264.

Rogers, R. D., \& Monsell, S. (1995). Costs of a predictable switch between simple cognitive tasks. Journal of Experimental Psychology: General, 124, 207-231.

Ruthruff, E., Remington, R. W., \& Johnston, J. C. (2001). Switching between simple cognitive tasks: The interaction of top-down and bottom-up factors. Journal of Experimental Psychology: Human Perception \& Performance, 27, 1404-1419.

ScHuch, S., \& Косн, I. (2003). The role of response selection for inhibition of task sets in task shifting. Journal of Experimental Psychology: Human Perception \& Performance, 29, 92-105.

Sohn, M.-H., \& Carlson, R. A. (2000). Effects of repetition and foreknowledge in task-set reconfiguration. Journal of Experimental Psychology: Learning, Memory, \& Cognition, 26, 1445-1460.

Spector, A., \& Biederman, I. (1976). Mental set and mental shift revisited. American Journal of Psychology, 89, 669-679.

WyLIE, G., \& AlLPORT, [D.] A. (2000). Task switching and the measurement of "switch costs." Psychological Research, 63, 212-233.

\section{NOTE}

1. We thank Mike Wendt for bringing this point to our attention.

(Manuscript received April 12, 2005; revision accepted for publication September 27, 2005.) 